$$
\begin{aligned}
& \therefore \quad \text { (n) } \\
& \text { Gnetiun hopliquées et fathématiques } \\
& \text { B-ajoO LIEGE }
\end{aligned}
$$

\title{
A CONFORMING FINITE ELEMENT FOR PLATE BENDING*
}

\author{
B. Fraeiss de VeubekE \\ Laboratory of Aerospace Engineering, University of Liège, Beigium
}

\begin{abstract}
A new, more compact, scheme is presented for building the $(16 \times 16)$ stiffness matrix of a finite plate element in bending. The element is of a conforming type: it satisfies continuity of deflections and slopes at the interiaces. In addition to the promise of good coinvergence characteristics, this property also guarantees lower bounds to infuence coefficients. The arbitrary quidrilateral shape is also convenient for versatility in the applications.
\end{abstract}

\section{INTRODUCTION}

THE difficulties involved in obtaining conforming displacement models for plate bending. have been stressed in the literature [1-3].

Continuity of deflections at the interfaces is the prime requirement to satisfy. The next requirement is the continuity of slopes which, if achieved, would produce a continuous, piecewise differential field of displacements in the structure. Most models, however, cannot comply rigorously with slope continuity. Although it appears from experience that local violations in slope continuity do not necessarily prevent convergence of deflections to the correct values when mesh sizes are reduced, the convergence of the stress field is much more seriously affected. Another reason for striving to a rigorous enforcement of slope continuity is the guarantee it provides that the direct influence coefficients obtained are actually lower bounds to the true ones. This, coupled with an analysis of the same problem using equilibrium models, opens the possibility to compare those lower bounds with upper bounds and so ascertain the value of the approximations $[4,5]$.

A conforming element of quadrilateral shape can be obtained from a combination of cubic deflection fields in the four triangular regions delimited by the edges of the quadrilateral and its diagonals. The principle of this construction and the advantage of using the diagonals as a natural oblique co-ordinate system for assembling the fields were the subject of earlier publications $[6,7]$. Further simplifications, reducing computer time, were discovered while setting up a program for the stiffness matrix of the element; they are reported here.

\section{STRAIN ENERGY OF A TRIANGULAR FIELD}

Consider the complete cubic deflection field

$$
w=\alpha_{1}+\alpha_{2} x+\alpha_{3} y+\alpha_{4} x^{2}+2 \alpha_{5} x y+\alpha_{6} y^{2}+4\left(\alpha_{7} x^{3}+\alpha_{8} x^{2} y+\alpha_{9} x y^{2}+\alpha_{10} y^{3}\right)
$$

valid in the triangular region 1 of Fig. 1.

* This research has been sponsored by the Air Force Flight Dynamics Laboratory under Contract AF 61(052)-892 through the European Office of Aerospace Research (EOAR), United States Air Force. 


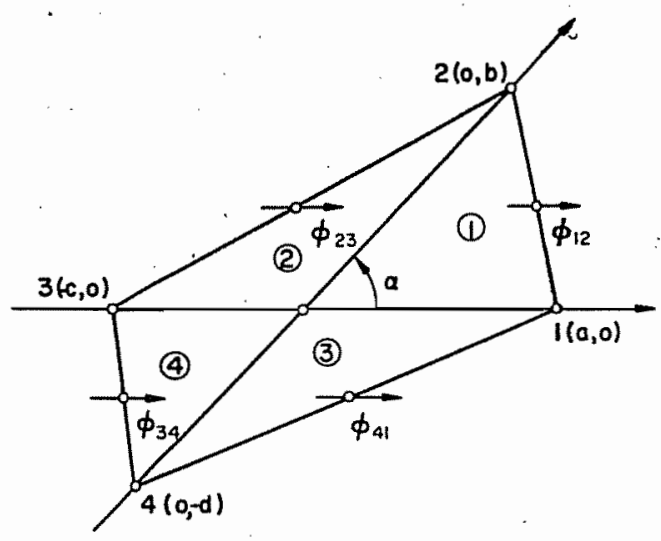

Fig. 1

The ten coefficients can be determined in terms of the local deflections $\left(w_{0}, w_{1}, w_{2}\right)$ at the nodes $1,2,0$, the local slopes $\left(\phi_{0}, \phi_{1}, \phi_{2}\right)$, where $\phi=\partial w / \partial x$, the local slopes $\left(\psi_{0}, \psi_{1}, \psi_{2}\right)$, where $\psi=\partial w / \partial y$, and the local slope $\phi_{12}$ at mid distance along the edge 12 .

$$
\begin{aligned}
\alpha_{1}=w_{0} \quad \alpha_{2}=\phi_{0} \quad \alpha_{3}=\psi_{0} \\
a^{2} \alpha_{4}=-3 w_{0}-2 a \phi_{0}+3 w_{1}-a \phi_{1} \\
2 a b \alpha_{5}=-6 w_{0}-2 a \phi_{0}-2 b \psi_{0}+6 w_{1}-a \phi_{1}+2 b \psi_{1}+a \phi_{2}-4 a \phi_{12} \\
b^{2} \alpha_{6}=-3 w_{0}-2 b \psi_{0}+3 w_{2}-b \psi_{2} \\
4 a^{3} \alpha_{7}=2 w_{0}+a \phi_{0}-2 w_{1}+a \phi_{1} \\
4 a^{2} b \alpha_{8}=6 w_{0}+2 a \phi_{0}+b \psi_{0}-6 w_{1}+a \phi_{1}-b \psi_{1}-a \phi_{2}+4 a \phi_{12} \\
4 a b^{2} \alpha_{9}=6 w_{0}+a \phi_{0}+2 b \psi_{0}-6 w_{1}+a \phi_{1}-2 b \psi_{1}+4 a \phi_{12} \\
4 b^{3} \alpha_{10}=2 w_{0}+b \psi_{0}-2 w_{2}+b \psi_{2}
\end{aligned}
$$

The bending strain energy in the oblique co-ordinates $(x, y)$ is

$$
U_{1}=\frac{1}{2 \sin \alpha} \iint_{1} D\left[\left(\frac{w_{x x}+w_{y y}-2 w_{x y} \cos \alpha}{\sin \alpha}\right)^{2}-2(1-v)\left(w_{x x} w_{y y}-w_{x y}^{2}\right)\right] \mathrm{d} x \mathrm{~d} y
$$

where $D$ is the bending rigidity of the plate. To calculate it in terms of the generalized coordinates

$$
\left(w_{0} \phi_{0} \psi_{0} w_{1} \phi_{1} \psi_{1} w_{2} \phi_{2} \psi_{2} \phi_{12}\right)=q_{1}^{\prime}
$$

( $q_{1}^{\prime}$ is the transpose of the column matrix $q_{1}$ of the generalized co-ordinates), we express the curvature as

$$
\gamma=\left(\begin{array}{c}
w_{x x} \\
w_{x y} \\
w_{y y}
\end{array}\right)=\left(\frac{1}{a^{2} b^{2}} W+\frac{2 x}{a^{3} b^{2}} W_{x}+\frac{2 y}{a^{2} b^{3}} W_{y}\right) q
$$


where the $(3 \times 10)$ matrices of constants $W, W_{x}$ and $W_{y}$ can be written down from

$$
\begin{aligned}
& w_{x x}=2 \alpha_{4}+24 x \alpha_{7}+8 y \alpha_{8} \\
& w_{x y}=2 \alpha_{5}+8 x \alpha_{8}+8 y \alpha_{9} \\
& w_{y y}=2 \alpha_{6}+8 x \alpha_{9}+24 y \alpha_{10}
\end{aligned}
$$

and substitution of the $\alpha_{i}$ from equations (2) and (3). Then

$$
U_{1}=\frac{1}{2} \iint_{1} D \gamma^{\prime} H y \mathrm{~d} x \mathrm{~d} y
$$

with

$$
H=\frac{1}{\sin ^{3} \alpha}\left(\begin{array}{ccc}
1 & -2 \cos \alpha & \cos ^{2} \alpha+v \sin ^{2} \alpha \\
-2 \cos \alpha & 4 \cos ^{2} \alpha+2(1-v) \sin ^{2} \alpha & -2 \cos \alpha \\
\cos ^{2} \alpha+\nu \sin ^{2} \alpha & -2 \cos \alpha & 1
\end{array}\right)
$$

- If the bending rigidity remains constant over the area of the triangle, equation (5) can be evaluated in terms of

$$
\begin{array}{clrl}
\iint_{1} D \mathrm{~d} x \mathrm{~d} y=\frac{a b}{2} D & \iint_{1} D x \mathrm{~d} x \mathrm{~d} y=\frac{a^{2} b}{6} D & \iint_{1} D y \mathrm{~d} x \mathrm{~d} y=\frac{a b^{2}}{6} D \\
\iint_{1} D x^{2} \mathrm{~d} x \mathrm{~d} y=\frac{a^{3} b}{12} D & \iint_{1} D x y \mathrm{~d} x \mathrm{~d} y=\frac{a^{2} b^{2}}{24} D & \iint_{1} D y^{2} \mathrm{~d} x \mathrm{~d} y=\frac{a b^{3}}{12} D .
\end{array}
$$

Then, having substituted equation (4) into (5)

$$
U_{1}=\frac{1}{2} q_{1}^{\prime} K_{1} q_{1}
$$

and the stiffness matrix of the first triangle can be calculated from

$$
\begin{aligned}
\frac{6 a^{3} b^{3}}{D} K_{1}= & 3 W^{\prime} H W+2\left(W_{x}^{\prime} H W+W^{\prime} H W_{x}+W_{y}^{\prime} H W+W^{\prime} H W_{y}^{\prime}+W_{x}^{\prime} H W_{x}+W_{y}^{\prime} H W_{y}\right) \\
& +W_{x}^{\prime} H W_{y}+W_{y}^{\prime} H W_{x} .
\end{aligned}
$$

Closer inspection of this formula reveals that, introducing the combinations

$$
A_{1}=W+W_{x} \quad B_{1}=W+W_{y} \quad C_{1}=W+W_{x}+W_{y}
$$

the number of products can be reduced to three:

$$
\frac{6 a^{3} b^{3}}{D} K_{1}=A_{1}^{\prime} H A_{1}+B_{1}^{\prime} H B_{1}+C_{1}^{\prime} H C_{1}
$$

Furthermore the matrices $A_{1}, B_{1}$ and $C_{1}$ are simpler.

$$
A_{1}=\left(\begin{array}{cccccccccc}
0 & -a b^{2} & 0 & 0 & a b^{2} & 0 & 0 & 0 & 0 & 0 \\
0 & 0 & -a b^{2} & 0 & 0 & a b^{2} & 0 & 0 & 0 & 0 \\
0 & a^{3} & -2 a^{2} b & -6 a^{2} & a^{3} & -2 a^{2} b & 6 a^{2} & 0 & -2 a^{2} b & 4 a^{3}
\end{array}\right)
$$




$$
\begin{aligned}
& B_{1}=\left(\begin{array}{cccccccccc}
0 & -2 a b^{2} & b^{3} & 0 & -a b^{2} & -b^{3} & 0 & -a b^{2} & 0 & 4 a b^{2} \\
0 & -a^{2} b & 0 & 0 & 0 & 0 & 0 & a^{2} b & 0 & 0 \\
0 & 0 & -a^{2} b & 0 & 0 & 0 & 0 & 0 & a^{2} b & 0
\end{array}\right) \\
& C_{1}=\left(\begin{array}{cccccccccc}
6 b^{2} & a b^{2} & b^{3} & -6 b^{2} & 2 a b^{2} & -b^{3} & 0 & -a b^{2} & 0 & 4 a b^{2} \\
6 a b & a^{2} b & a b^{2} & -6 a b & a^{2} b & -a b^{2} & 0 & 0 & 0 & 4 a^{2} b \\
6 a^{2} & a^{3} & a^{2} b & -6 a^{2} & a^{3} & -2 a^{2} b & 0 & 0 & a^{2} b & 4 a^{3}
\end{array}\right)
\end{aligned}
$$

\section{ASSEMBLING THE STIFFNESS MATRICES OF THE TRIANGULAR FIELDS}

In the second triangular field we can obtain continuity along the interface $x=0$ of the deflection $w$ (hence also continuity of $\partial w / \partial y$ ) and of the slope $\partial w / \partial x$ by retaining the values of the coefficients $\alpha_{1}, \alpha_{2}, \alpha_{3}, \alpha_{5}, \alpha_{6}, \alpha_{9}$ and $\alpha_{10}$ of the first field.

New values $\alpha_{4}^{\prime}, \alpha_{7}^{\prime}$ and $\alpha_{8}^{\prime}$ can be adopted for the remaining coefficients. It can be verified that, as would be expected, the coefficients of the second field are given by the same formulas (2) and (3), provided we change $a$ into $-c, w_{1}$ into $w_{3}, \phi_{1}$ into $\phi_{3}, \psi_{1}$ into $\psi_{3}$, $\phi_{12}$ into $\phi_{23}$. The generalized co-ordinates for the second field are thus

$$
\left(w_{0} \phi_{0} \psi_{0} w_{3} \phi_{3} \psi_{3} w_{2} \phi_{2} \psi_{2} \phi_{23}\right)=q_{2}^{\prime} \text {. }
$$

Proceeding in the same manner as for the first field, the strain energy

$$
U_{2}=\frac{1}{2} q_{2}^{\prime} K_{2} q_{2}
$$

produces a stiffness matrix $K_{2}$ given by

$$
\frac{6 c^{3} b^{3}}{D} K_{2}=A_{2}^{\prime} H A_{2}+B_{2}^{\prime} H B_{2}+C_{2}^{\prime} H C_{2}
$$

where $A_{2}, B_{2}$ and $C_{2}$ are directly obtainable from the corresponding matrices (11), (12) and (13) of the first field by changing $a$ into $-c$.

For field 3, deflections and slopes at the interface $y=0$ are identical with those of field 1 if the coefficients $\alpha_{1}, \alpha_{2}, \alpha_{3}, \alpha_{4}, \alpha_{5}, \alpha_{7}$ and $\alpha_{8}$ are retained.

The new coefficients are $\alpha_{6}^{\prime}, \alpha_{9}^{\prime}$ and $\alpha_{10}^{\prime}$. To determine the coefficients in terms of the generalized displacements

$$
\left(w_{0} \phi_{0} \psi_{0} w_{1} \phi_{1} \psi_{1} w_{4} \phi_{4} \psi_{4} \phi_{41}\right)=q_{3}^{\prime}
$$

we can use formulas (2) and (3) with the modifications $b$ into $-d, w_{2}$ into $w_{4}, \phi_{2}$ into $\phi_{4}$, $\psi_{2}$ into $\psi_{4}$ and $\phi_{12}$ into $\phi_{41}$. The stiffness matrix $K_{3}$ is given by

$$
\frac{6 a^{3} d^{3}}{D} K_{3}=A_{3}^{\prime} H A_{3}+B_{3}^{\prime} H B_{3}+C_{3}^{\prime} H C_{3}
$$

where $A_{3}, B_{3}, C_{3}$ derive from $A_{1}, B_{1}, C_{1}$ by changing $b$ into $-d$. Finally the fourth field conforms with fields 2 and 3 at the interfaces, if its coefficients are

$$
\alpha_{1}, \alpha_{2}, \alpha_{3}, \alpha_{4}^{\prime}, \alpha_{5}, \alpha_{6}^{\prime}, \alpha_{7}^{\prime}, \alpha_{8}^{\prime}, \alpha_{9}^{\prime} \text { and } \alpha_{10}^{\prime}
$$


In terms of the generalized co-ordinates

$$
\left(w_{0} \phi_{0} \psi_{0} w_{3} \phi_{3} \psi_{3} w_{4} \phi_{4} \psi_{4} \phi_{34}\right)=q_{4}^{\prime}
$$

the coefficients derive from equations (2) and (3) with the combined modifications: $a$ into $-c, b$ into $-d$

$$
\begin{array}{ccc}
w_{1}, \phi_{1}, \psi_{1} & \text { respectively into } & w_{3}, \phi_{3}, \psi_{3} \\
w_{2}, \phi_{2}, \psi_{2} & \text { respectively into } w_{4}, \phi_{4}, \psi_{4} \\
\phi_{12} \text { into } \phi_{34} .
\end{array}
$$

Again, with the double change : $a$ into $-c$ and $b$ into $-d$, the matrices (11)-(13) can be used to form

$$
\frac{6 c^{3} d^{3}}{D} K_{4}=A_{4}^{\prime} H A_{4}+B_{4}^{\prime} H B_{4}+C_{4}^{\prime} H C_{4}
$$

The four partial stiffness matrices are combined into a $(19 \times 19)$ matrix $J$ defined by energy addition

$$
\sum_{1}^{4} q_{i}^{\prime} K_{i} q_{i}=p^{\prime} J p
$$

where

$$
p^{\prime}=\left(w_{0} \phi_{0} \psi_{0} w_{1} \phi_{1} \psi_{1} w_{2} \phi_{2} \psi_{2} w_{3} \phi_{3} \psi_{3} w_{4} \phi_{4} \psi_{4} \phi_{12} \phi_{23} \phi_{34} \phi_{41}\right)
$$

This can be done by using localizing matrices $L_{i}$

$$
\begin{aligned}
q_{i} & =L_{i} p \\
J & =\sum_{1}^{4} L_{i}^{\prime} K_{i} L_{i}
\end{aligned}
$$

or simply addressing directly the elements to their proper place in $J$ as they are generated.

\section{CONDENSATION}

$J$ is not a proper stiffness matrix since the nineteen co-ordinates in $p$ are not independent. They depend on sixteen coefficients

$$
\alpha_{i}(i=1,2,3 \ldots 10) \text { and } \alpha_{i}^{\prime}(i=4,6,7,8,9,10)
$$

It is interesting to observe that $\alpha_{1}, \alpha_{2}, \alpha_{3}$ which represent the rigid body freedoms of the plate and $\alpha_{5}$, which represents a torsional deformation mode, are the only coefficients valid throughout the four fields. Equating the four different field expressions obtained from equation (3) for coefficient $\alpha_{5}$, we obtain a system of three equations that can easily 
be solved for the deflection and slope at the internal node:

$$
\begin{aligned}
w_{0}= & \frac{c}{a+c} w_{1}+\frac{a}{a+c} w_{3}-\frac{a c}{6(a+c)} \phi_{1}+\frac{a c}{6(a+c)} \phi_{3} \\
& +\frac{2 a c}{3(a+c)(b+d)}\left(-d \phi_{12}+d \phi_{23}+b \phi_{34}-b \phi_{41}\right) \\
\phi_{0}= & \frac{3}{a+c}\left(w_{1}-w_{3}\right)-\frac{a}{2(a+c)} \phi_{1}-\frac{c}{2(a+c)} \phi_{3}+\frac{d}{2(b+d)} \phi_{2}+\frac{b}{2(b+d)} \phi_{4} \\
& -\frac{2}{(a+c)(b+d)}\left(a d \phi_{12}+c d \phi_{23}+b c \phi_{34}+a b \phi_{41}\right) \\
\psi_{0}= & \frac{c}{a+c} \psi_{1}+\frac{a}{a+c} \psi_{3}+\frac{2 a c}{(a+c)(b+d)}\left(-\phi_{12}+\phi_{23}-\phi_{34}+\phi_{41}\right) .
\end{aligned}
$$

It can be verified that, with these values of $w_{0}, \phi_{0}$ and $\psi_{0}$, the other equations between different expressions of the $\alpha_{i}$ and $\alpha_{i}^{\prime}$ coefficients turn into identities. Hence the sixteen coefficients can be expressed in terms of the sixteen generalized co-ordinates

$$
\left(w_{1} \phi_{1} \psi_{1} w_{2} \phi_{2} \psi_{2} w_{3} \phi_{3} \psi_{3} w_{4} \phi_{4} \psi_{4} \phi_{12} \phi_{23} \phi_{34} \phi_{41}\right)=q^{\prime}
$$

by a non-singular transformation.

If the linear relations (17) are put in matrix form

$$
\left(\begin{array}{l}
w_{0} \\
\phi_{0} \\
\psi_{0}
\end{array}\right)=M q
$$

a condensation matrix $N$ is defined by

$$
N=\left(\begin{array}{l}
M \\
E
\end{array}\right)
$$

where $E$ is a $(16 \times 16)$ identity matrix, so that

$$
p=N q .
$$

The total energy becomes

$$
\frac{1}{2} q^{\prime} K q
$$

with the (proper) stiffness matrix

$$
K=N^{\prime} J N
$$

\section{NORMALIZATION TO COMMON REFERENCE DIRECTIONS}

$K$ is now the stiffness matrix of a conforming element.

Indeed, consider the edge 12 and suppose the quantities $\left(w_{1}, \phi_{1}, \psi_{1}\right)\left(w_{2}, \phi_{2}, \psi_{2}\right)$ and $\phi_{12}$ to be known. From $\phi_{1}$ and $\psi_{1}$ we can deduce the slopes $s_{1}$ and $n_{1}$ respectively in the direction 12 and normal to it in 1 . Similarly from $\phi_{2}$ and $\psi_{2}$ we can deduce $s_{2}$ and $n_{2}$. The 
knowledge of $\left(w_{1}, w_{2}, s_{1}, s_{2}\right)$ is just sufficient to determine $w$ everywhere, since it varies as a cubic along the edge. In particular the slope $s_{12}$ at mid distance is thereby determined. From $s_{12}$ and $\phi_{1.2}, n_{12}$ can now be determined and the knowledge of $n_{1}, n_{2}$ and $n_{12}$ is just sufficient to determine the transverse slope $n$ everywhere, since it has parabolic variation. Consequently, the knowledge of $\left(w_{1}, \phi_{1}, \psi_{1}\right),\left(w_{2}, \phi_{2}, \psi_{2}\right)$ and $\phi_{12}$ determines completely the defiections and slopes along the edge 12 . Should this edge become an interface, continuity of deflections and slopes will follow from the single-valuedness of deflections and slopes in the nodes 1 and 2 and single-valuedness of the transverse slope at mid distance.

As the natural reference directions, provided by the diagonals, can change when passing from one element to the other, it is advisable to refer the slopes to common reference directions. This facilitates pairwise identification of the co-ordinates that will in fact become the nodal displacements of the assembled structure.

For the identification of slopes at mid distance of an edge the best policy is to use as generalized co-ordinates the slopes in the directions of the outward normal (Fig. 2).

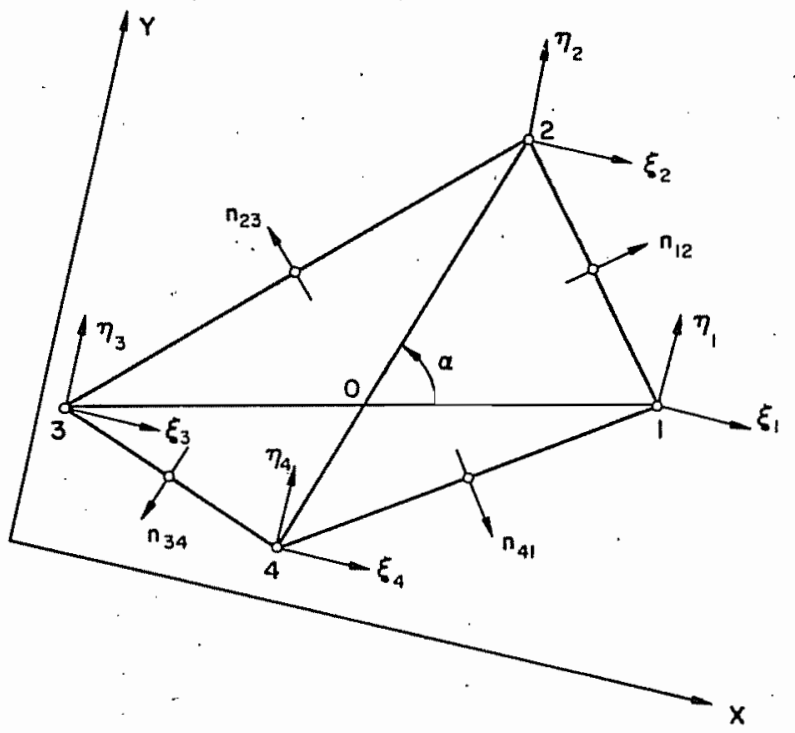

FiG. 2

Then, when assembling elements, account must be taken of the reversal of sign of the reference direction. Taking the case of the edge 12, the problem consists in expressing $\phi_{12}$ in terms of the slope $n_{12}$ in the direction of the outward normal and $\left(w_{1}, \phi_{1}, \psi_{1}\right)$, $\left(w_{2}, \phi_{2}, \psi_{2}\right)$.

One procedure is as follows. From $\phi_{1}$ and $\psi_{1}$, deduce the slope $s_{1}$ in the direction 12

$$
\begin{aligned}
c_{12} s_{1} & =-a \phi_{1}+b \psi_{1} \\
c_{12} & =\left(a^{2}+b^{2}-2 a b \cos \alpha\right)^{\frac{1}{2}} \text { the length of the edge. }
\end{aligned}
$$

Similarly in 2

$$
c_{12} s_{2}=-a \phi_{2}+b \psi_{2}
$$


Then, considering the cubic variation of $w$ along 12, express the slope $s_{12}$ at mid distance

$$
4 c_{12} s_{12}=6 w_{2}-6 w_{1}-c_{12}\left(s_{1}+s_{2}\right)
$$

or, substituting $s_{1}$ and $s_{2}$,

$$
4 c_{12} s_{12}=6 w_{2}-6 w_{1}+a \phi_{1}-b \psi_{1}+a \phi_{2}-b \psi_{2} .
$$

Finally express $\phi_{12}$ in terms of $s_{12}$ and $n_{12}$

$$
c_{12} \phi_{12}=b \sin \alpha n_{12}-(a-b \cos \alpha) s_{12} \text {. }
$$

When $s_{12}$ is substituted the required formula is

$$
\phi_{12}=\frac{b \sin \alpha}{c_{12}} n_{12}-\frac{a-b \cos \alpha}{4 c_{12}^{2}}\left(6 w_{2}-6 w_{1}+a \phi_{1}-b \psi_{1}+a \phi_{2}-b \psi_{2}\right) .
$$

At the vertices, where several elements are interconnected, the slopes should be referred to a common cartesian reference frame (Fig. 2). The cartesian slopes $\partial w / \partial X$ and $\partial w / \partial Y$ will be denoted by $\xi$ and $\eta$ respectively. They are related to the slopes $\phi$ and $\psi$ in the reference frame of the diagonals by

$$
\phi=\xi \frac{\partial X}{\partial x}+\eta \frac{\partial Y}{\partial x} \quad \psi=\xi \frac{\partial X}{\partial y}+\eta \frac{\partial Y}{\partial y}
$$

There is a choice for expressing the direction cosines, which can sometimes produce analytical simplifications:

$$
\begin{aligned}
& \frac{\partial X}{\partial x}=\frac{x_{10}}{a}=\frac{x_{03}}{c}=\frac{x_{13}}{a+c} \\
& \frac{\partial Y}{\partial x}=\frac{y_{10}}{a}=\frac{y_{03}}{c}=\frac{y_{13}}{a+c} \\
& \frac{\partial X}{\partial y}=\frac{x_{20}}{b}=\frac{x_{04}}{d}=\frac{x_{24}}{b+d} \\
& \frac{\partial Y}{\partial y}=\frac{y_{20}}{b}=\frac{y_{04}}{d}=\frac{y_{24}}{b+d} .
\end{aligned}
$$

In these formulas $x_{i j}$ stands for $x_{i}-x_{j}$ and $y_{i j}$ for $y_{i}-y_{j}$, where $\left(x_{i}, y_{i}\right)$ are the co-ordinates of the intersection of the diagonals $(i=0)$ and of the vertices $(i=1,2,3,4)$ in the cartesian reference frame. The computer can generate the required geometrical characteristics from the co-ordinates of the vertices alone by formulas such as

$$
\begin{aligned}
& x_{0}=\frac{x_{1} x_{2} y_{34}+x_{2} x_{3} y_{41}+x_{3} x_{4} y_{12}+x_{4} x_{1} y_{23}}{y_{31} x_{24}-x_{31} y_{24}} \\
& y_{0}=\frac{y_{1} y_{2} x_{34}+y_{2} y_{3} x_{41}+y_{3} y_{4} x_{12}+y_{4} y_{1} x_{23}}{x_{31} y_{24}-y_{31} x_{24}}
\end{aligned}
$$




$$
\begin{aligned}
a^{2} & =x_{10}^{2}+y_{10}^{2} \\
(a+c)^{2} & =x_{13}^{2}+y_{13}^{2} \\
c_{12}^{2} & =x_{12}^{2}+y_{12}^{2} \\
a b \sin \alpha & =x_{10} y_{20}-y_{10} x_{20} \\
a b \cos \alpha & =x_{10} x_{20}+y_{10} y_{20} \\
a(a-b \cos \alpha) & =x_{10} x_{12}+y_{10} y_{12}
\end{aligned}
$$

Our final (conforming) set of generalized co-ordinates is described by the row vector

$$
r^{\prime}=\left(w_{1} \eta_{1} \xi_{1} w_{2} \eta_{2} \xi_{2} w_{3} \eta_{3} \xi_{3} w_{4} \eta_{4} \xi_{4} n_{12} n_{23} n_{34} n_{41}\right)
$$

The transformation

$$
q=\operatorname{Pr}
$$

from the old (natural) set of co-ordinates to the conforming one is made up from eight equations of type (24) applied to the slopes at the vertices, four identities $w_{i}=w_{i}$ at the vertices, and four equations of type (23) in which, however, the natural slopes at the vertices are still to be replaced by the cartesian ones. The result for $\phi_{12}$ turns out to be

$$
\begin{aligned}
\phi_{12}= & \frac{x_{10} y_{20}-y_{10} x_{20}}{\sqrt{ }\left[\left(x_{10}^{2}+y_{10}^{2}\right)\left(x_{12}^{2}+y_{12}^{2}\right)\right]} n_{12} \\
& -\frac{x_{10} x_{12}+y_{10} y_{12}}{4\left(x_{12}^{2}+y_{12}^{2}\right) \sqrt{\left(x_{10}^{2}+y_{10}^{2}\right)}}\left(6 w_{2}-6 w_{1}+x_{12} \xi_{1}+x_{12} \xi_{2}+y_{12} \eta_{1}+y_{12} \eta_{2}\right) .
\end{aligned}
$$

In this form, the other $\phi_{i j}$ values can be obtained by a cyclic permutation of the indices $(1,2,3,4)$.

Under the linear transformation (28) the energy of the element becomes the quadratic form

$$
\frac{1}{2} r^{\prime} R r
$$

in the conforming co-ordinates. The final (operational) stiffness matrix $R$ is to be calculated from

$$
R=P^{\prime} K P \quad \text { or } \quad R=Q^{\prime} J Q
$$

where $Q=N P$ is the product of the condensing matrix $N$ and the conforming matrix $P$. This product can be programmed numerically. If an analytical form is preferred, the only algebra remaining is the substitution of equations (24) and (29) into equations (17).

\section{SHAPING OR WEIGHTING FUNCTIONS}

In order to translate externally applied loads into generalized loads, the transverse displacement of the plate should be expressed in terms of the generalized displacements. Suppose that in each field $(i=1,2,3,4) w$ is expressed in function of the co-ordinates $q_{i}$. 
For example, in field 1,

$$
\begin{aligned}
w= & w_{0} W_{0,1}(x, y)+\phi_{0} \Phi_{0,1}(x, y)+\psi_{0} \Psi_{0,1}(x, y)+w_{1} W_{1,1}(x, y) \\
& +\phi_{1} \Phi_{1,1}(x, y)+\psi_{1} \Psi_{1,1}(x, y)+w_{2} W_{2,1}(x, y)+\phi_{2} \Phi_{2,1}(x, y) \\
& +\psi_{2} \Psi_{2,1}(x, y)+\phi_{12} \Phi_{12}(x, y) .
\end{aligned}
$$

Then, the virtual work equation for a transverse load $\pi(x, y)$ on that part of the element is

$$
\sin \alpha \iint_{1} \pi(x, y) w \mathrm{~d} x \mathrm{~d} y=q_{1}^{\prime} f_{1}
$$

where the elements of the generalized loads matrix $f_{1}$ are in succession

$$
\sin \alpha \iint_{1} \pi(x, y) W_{0,1}^{\gamma}(x, y) \mathrm{d} x \mathrm{~d} y, \sin \alpha \iint_{1} \pi(x, y) \Phi_{0,1}(x, y) \mathrm{d} x \mathrm{~d} y, \text { etc. }
$$

The contribution of concentrated loads is simply obtained by multiplication of the load by the local values of the weighting functions

$$
W_{0,1}(x, y), \text { etc. }
$$

The total virtual work, adding the four field contributions, will be, by virtue of equation (15)

$$
\sum_{1}^{4} q_{i}^{\prime} f_{i}=p^{\prime} \sum_{1}^{4} L_{i}^{\prime} f_{i}=p^{\prime} f
$$

Hence, the generalized loads conjugate to the generalized displacements in $p$ are obtained from

$$
f=\sum_{1}^{4} L_{i}^{\prime} f_{i}
$$

Again, from the invariance of the virtual work, and by virtue of relations (20), (28) and (30)

$$
g=N^{\prime}\left(\sum_{1}^{4} L_{i}^{\prime} f_{i}\right) \quad\left(\text { virtual work } g^{\prime} q\right)
$$

is the loads matrix conjugate to $q$ (defining a set of "natural" generalized displacements), while

$$
h=P^{\prime} g=Q^{\prime}\left(\sum_{1}^{4} L_{i}^{\prime} f_{i}\right) \quad\left(\text { virtual work } h^{\prime} r\right)
$$

is the loads matrix conjugate to $r$ (defining a set of "conforming" generalized displacements).

Hence, since the matrix $Q$ is available, being necessary to generate the operational stiffness matrix, it is convenient to evaluate the loads by the $f_{i}$ contributions.

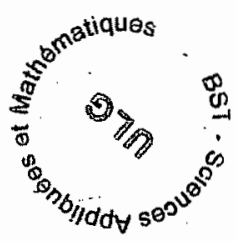


The required weighting functions for field 1 turn out to be:

$$
\begin{aligned}
& W_{0,1}=\left(1-\frac{x}{a}-\frac{y}{b}\right)^{2}\left(1+2 \frac{x}{a}+2 \frac{y}{b}\right) \\
& \Phi_{0,1}=x\left(1-\frac{x}{a}-\frac{y}{b}\right)^{2} \\
& \Psi_{0,1}=y\left(1-\frac{x}{a}-\frac{y}{b}\right)^{2} \\
& W_{1,1}=\frac{x}{a}\left(3 \frac{x}{a}+6 \frac{y}{b}-2 \frac{x^{2}}{a^{2}}-6 \frac{x}{a} \frac{y}{b}-6 \frac{y^{2}}{b^{2}}\right) \\
& \Phi_{1,1}=x\left(-\frac{x}{a}-\frac{y}{b}+\frac{x^{2}}{a^{2}}+\frac{x}{a} \frac{y}{b}+\frac{y^{2}}{b^{2}}\right) \\
& \Psi_{1,1}=y \frac{x}{a}\left(2-\frac{x}{a}-2 \frac{y}{b}\right) \\
& W_{2,1}=\frac{y^{2}}{b^{2}}\left(3-2 \frac{y}{b}\right) \\
& \Phi_{2,1}=x \frac{y}{b}\left(1-\frac{x}{a}\right) \\
& \Phi_{2,1}=y \frac{y}{b}\left(-1+\frac{y}{b}\right) \\
& =4 x \frac{y}{b}\left(-1+\frac{x}{a}+\frac{y}{b}\right)
\end{aligned}
$$

The modifications to introduce in order to convert those functions into the weighting functions of fields 2, 3 and 4 are exactly.the same as those discussed in Section 3.

\section{GENERALIZED EXTERNAL LOADS FOR UNIFORM TRANSVERSE LOADING}

To evaluate the integrals involved in equation (32), which are extended over a triangular field, it is convenient to change variables as follows:

$$
\frac{x}{a}=v-u \quad \frac{y}{b}=1-v
$$

so that equation (32) is transformed into

$$
q_{1}^{\prime} f_{1}=a b \sin \alpha \int_{0}^{1}\left\{\int_{0}^{v} \pi(x, y) w \mathrm{~d} u\right\} \mathrm{d} v
$$


and the weighting functions (37) become

$$
\begin{aligned}
& W_{0,1}=u^{2}(3-2 u) \\
& \Phi_{0,1}=a u^{2}(v-u) \\
& \Psi_{0,1}=b u^{2}(1-v) \\
& W_{1,1}=v^{2}(3-2 v)-u^{2}(3-2 u) \\
& \Phi_{1,1}=a(v-u)\left(u^{2}+v^{2}-u v-v\right) \\
& \Psi_{1,1}=b\left(v^{2}-u^{2}\right)(1-v) \\
& W_{2,1}=(1-v)^{2}(1+2 v) \\
& \Phi_{2,1}=a(v-u)(1-v)(1-v+u) \\
& \Psi_{2,1}=-b v(1-v)^{2} \\
& \Phi_{12}=-4 a u(v-u)(1-v)
\end{aligned}
$$

In the case of a uniform transverse load $\pi_{1}$ in field one, integration produces the following generalized loads matrix

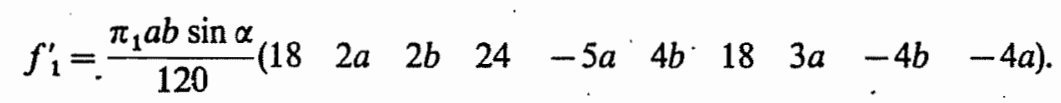

Similar results are immediately obtainable for the three other fields by the appropriate substitutions in the geometrical parameters $a$ and $b$. The proper combinations generating the equivalent external loads for the element are then obtained through equation (36).

\section{LAGRANGIAN MASS MATRICES}

Let $m_{1}(x, y)$ be the distribution of mass over the area of the first field of the plate element. The kinetic energy, neglecting the rotary inertia, will then be

$$
\sin \alpha \frac{1}{2} \iint_{1} m_{1}(x, y) \dot{w}^{2}(x, y) \mathrm{d} x \mathrm{~d} y=\frac{1}{2} a b \sin \alpha \int_{0}^{1}\left\{\int_{0}^{v} m_{1}(u, v) \dot{w}^{2}(u, v) \mathrm{d} u\right\} \mathrm{d} v .
$$

Substitution of the transverse velocity in terms of the shaping functions

$$
\dot{w}=\dot{w}_{0} W_{0,1}(u, v)+\cdots+\dot{\phi}_{12} \Phi_{12}(u, v)
$$

gives the quadratic form

$$
\frac{1}{2} \dot{q}_{1}^{\prime} M_{1} \dot{q}_{1}
$$

With a Lagrangian mass matrix $M_{1}$ having elements defined by

$$
\begin{aligned}
& a b \sin \alpha \int_{0}^{1}\left\{\int_{0}^{v} m_{1}(u, v) W_{0,1}^{2}(u, v) \mathrm{d} u\right\} \mathrm{d} v \\
& a b \sin \alpha \int_{0}^{1}\left\{\int_{0}^{v} m_{1}(u, v) W_{0,1} \Phi_{0,1} \mathrm{~d} u\right\} \mathrm{d} v \text { etc. }
\end{aligned}
$$


Those elements have been evaluated for the case of a constant mass distribution $m_{1}(x, y)=m$ and the resulting Lagrangian mass matrix $M_{1}$ is given in Table 1 . The corresponding matrices for the other fields follow by the appropriate modifications in the geometrical constants.

TABLE 1. LAGRANGIAN MASS MATRIX FOR THE $q_{1 .}$ CO-ORDINATES; UNIFORM MASS DISTRIBUTION $m$

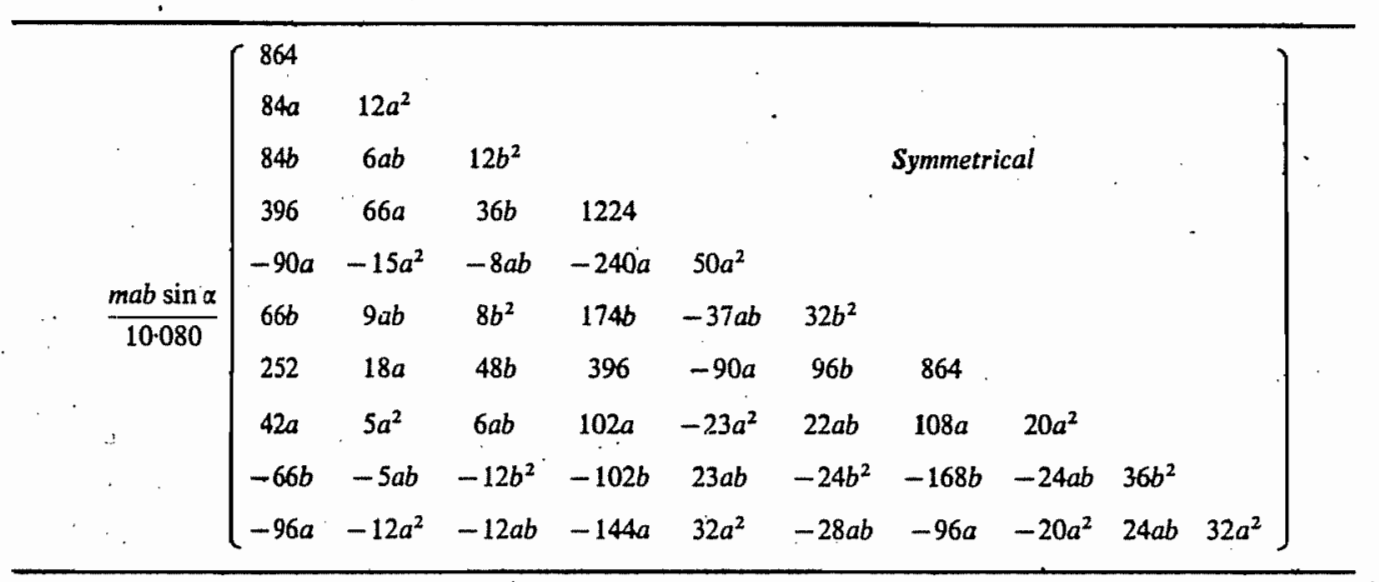

The Lagrangian mass matrix of the element in conforming co-ordinates follows by addition of energies and application of the linear transformations (15), (20) and (28) as

$$
M=P^{\prime} N^{\prime}\left(\sum_{1}^{4} L_{i}^{\prime} M_{i} L_{i}\right) N P=Q^{\prime}\left(\sum_{1}^{4} L_{i}^{\prime} M_{i} L_{i}\right) Q
$$

\section{FINAL REMARKS}

The element is now operational and preliminary results for the case of a rectangular plate, centrally loaded, under various boundary conditions, are extremely satisfactory from the deflections point of view. Numerical results and comparison with other reported data are given in [8].

Finally, in a private communication, Professor T. H. Pian pointed out the similarity of our technique for assembling polynomial approximations to the methods described in [9-11].

\section{REFERENCES}

[1] O. C. Zienkiewicz and Y. K. CheUnG, The finite element method for analysis of elastic isotropic and orthotropic slabs. Proc. Instn civ. Engrs 28, 471-488 (1964).

[2] G. P. Bazeley, Y. K. Cheung, B. M. Irons and O. C. Zienkiewicz, Triangular elements in bending. Conforming and non-conforming solutions. Proc. Conf. Matrix Methods in Structural Mechanics, Air Force Institute of Technology, Dayton, Ohio, Oct. (1965).

[3] R. W. Clough and J. L. Tocher, Finite element stiffness matrices for analysis of plates in bending. Proc. Conf. Matrix Methods in Structural Mechanics, Air Force Institute of Technology, Dayton, Ohio, Oct. (1965). 
[4] B. Fraeiss DE VeuBEKE, Upper and lower bounds in matrix structural analysis. AGARDograph 78, pp. 165-201. Pergamon Press (1963).

[5] B. FraEIJS DE VeubeKE, Displacement and equilibrium models in the finite element method. Stress Analysis, edited by O. C. ZiENKIEWICZ and G. S. Holister, Chapter 9. Wiley (1965).

[6] G. SANDER, Bornes supérieures et infërieures dans l'analyse matricielle des plaques en flexion-torsion. Bull. Soc. r. Sci. Liége 33, 456-494 (1964).

[7] B. Fraeus de Veubeke, Bending and stretching of plates. Proc. Conf. Matrix Methods in Structural Mechanics, Air Force Institute of Technology, Dayton, Ohio, Oct. (1965).

[8] G. SANDER and B. Fraeiss DE Veubeke, Upper and lower bounds to structural deflections by dual analysis in finite elements. Annual Summary I, Air Force Technical Report AFFDL-TR-66-199, pp. 3-21 to 3-27 (1966).

[9] G. Birkhoff and H. L. Garabedian, Smooth surface interpolation. J. Math. Phys. 39, 258-268 (1960).

[10] C. DE Boor, Bicubic spline interpolation. J. Math. Phys. 41, 212-218 (1962).

[11] G. BIRKHOFF and C. DE BoOR, Piecewise Polynomial Interpolation and Approximation. Elsevier (1965).

(Received 23 January 1967)

Абстракт-Предлагается для расчета сооружений новая, более компактная схема $(16 \times 16)$ матрицы коэффициентов жесткости для конечного изгибаемого элемента пластинки. Элемент является соответствующим типом так, как удовлетворяет условию непрерывности изгибов и имеет наклоны на поверхностях раздела. Добавочно, относительно перспективы надлежашей сходимости характеристик, такое свойство обеспечивает также низшие скачки, которые влияют на коэффициенты. Чтоже касается многосторонности метода, обычная четыреугольная форма является также удобной в применениях. 